\title{
1 A chromosome-level assembly of the black tiger shrimp (Penaeus monodon) genome facilitates
}

\section{2 the identification of novel growth-associated genes}

3

4 Tanaporn Uengwetwanit ${ }^{1, \uparrow}$, Wirulda Pootakham ${ }^{2, \dagger}$, Intawat Nookaew ${ }^{3}$, Chutima Sonthirod ${ }^{2}$,

5 Pacharaporn Angthong ${ }^{1}$, Kanchana Sittikankaew ${ }^{1}$, Wanilada Rungrassamee ${ }^{1}$, Sopacha

6 Arayamethakorn $^{1}$, Thidathip Wongsurawat ${ }^{3}$, Piroon Jenjaroenpun ${ }^{3}$, Duangjai Sangsrakru ${ }^{2}$, Rungnapa

7 Leelatanawit ${ }^{1}$, Jutatip Khudet ${ }^{4}$, Jasper J. Koehorst ${ }^{5}$, Peter J. Schaap ${ }^{5}$, Vitor Martins dos Santos ${ }^{5}$,

8 Frédéric Tangy ${ }^{6}$, Nitsara Karoonuthaisiri ${ }^{1}{ }^{1 *}$

9

$10{ }^{1}$ National Center for Genetic Engineering and Biotechnology (BIOTEC), National Science and

11 Technology Development Agency, Pathum Thani, 12120, Thailand

$12{ }^{2}$ National Omics Center, National Center for Genetic Engineering and Biotechnology (BIOTEC),

13 National Science and Technology Development Agency, Pathum Thani, 12120, Thailand

$14{ }^{3}$ Department of Biomedical Informatics, College of Medicine, University of Arkansas for Medical

15 Sciences, Little Rock, Arkansas, 72205, USA

$16{ }^{4}$ Shrimp Genetic Improvement Center, Integrative Aquaculture Biotechnology Research Group, Surat

17 Thani, 84110, Thailand

$18{ }^{5}$ Laboratory of Systems and Synthetic Biology, Department of Agrotechnology and Food Sciences,

19 Wageningen University and Research, 6708WE Wageningen, Netherland

$20{ }^{6}$ Viral Genomics and Vaccination Unit, UMR3569 CNRS, Virology department, Institut Pasteur,

2175015 , Paris, France

22

$23{ }^{\dagger}$ These authors contributed equally to this work

$24 *$ Correspondence should be addressed to NK (nitsara@alumni.stanford.edu) 
27 The black tiger shrimp (Penaeus monodon) is one of the most prominent farmed crustacean species

28 with an average annual global production of 0.5 million tons in the last decade. To ensure sustainable

29 and profitable production through genetic selective breeding programs, several research groups have

30 attempted to generate a reference genome using short-read sequencing technology. However, the

31 currently available assemblies lack the contiguity and completeness required for accurate genome

32 annotation due to the highly repetitive nature of the genome and technical difficulty in extracting high-

33 quality, high-molecular weight DNA in this species. Here, we report the first chromosome-level

34 whole-genome assembly of P. monodon. The combination of long-read Pacific Biosciences (PacBio)

35 and long-range Chicago and Hi-C technologies enabled a successful assembly of this first high-quality

36 genome sequence. The final assembly covered $2.39 \mathrm{~Gb}(92.3 \%$ of the estimated genome size) and

37 contained 44 pseudomolecules, corresponding to the haploid chromosome number. Repetitive

38 elements occupied a substantial portion of the assembly (62.5\%), highest of the figures reported

39 among crustacean species. The availability of this high-quality genome assembly enabled the

40 identification of novel genes associated with rapid growth in the black tiger shrimp through the

41 comparison of hepatopancreas transcriptome of slow-growing and fast-growing shrimps. The results

42 highlighted several gene groups involved in nutrient metabolism pathways and revealed 67 newly

43 identified growth-associated genes. Our high-quality genome assembly provides an invaluable

44 resource for accelerating the development of improved shrimp strain in breeding programs and future

45 studies on gene regulations and comparative genomics.

46

\section{Keywords}

48 Black tiger shrimp; Penaeus monodon; reference genome; Hi-C; PacBio; growth-associated

49 genes; transcriptomics

50

\section{Introduction}


52 Aquaculture is an important food source for the world's growing population as it relieves the

53 over-consumption pressure of natural animal populations within the aquatic environment. Currently,

54 aquaculture provides the planet with more than 50 percent of fish products consumed by humans,

55 making it one of the world's fastest-growing food sectors with an annual growth rate of 5.8 percent

56 since 2010 (FAO, 2018). The penaeid marine shrimps (Family Penaeidae) are the predominately

57 cultured group (Thornber et al., 2019), with an annual production exceeding 4.5 million tons

58 (Anderson, 2019). In this group, the Pacific white shrimp (Litopenaeus vannamei) and black tiger

59 shrimp (Penaeus monodon) are the most dominant species cultured, accounting for $53 \%$ and $9 \%$ of

60 total crustacean production, respectively (FAO, 2018).

61 While the penaeid industry has seen dramatic growth for the past few decades, industrial

62 production of $P$. monodon proved to be unsustainable due to a lack of biological and genetic

63 knowledge to achieve its desirable traits such as fast growth, disease resistance, reproductive

64 maturation without reliance on wild brooders (Guppy et al., 2018). Recently, the L. vannamei breeding

65 and domestication programs can be expeditely improved due to the available genome sequence, which

66 allows selective breeding and helps in overcoming industrial challenges (Zhang et al., 2019).

67 Genomic-driven breeding and domestication programs for P. monodon, on the other hand, are still in

68 their infancy due to the absense of an informative high-quality draft genome sequence.

70 efforts have been made to investigate the genome and genetic architecture of this important species

71 over the past few decades. BAC library construction (Wuthisuthimethavee, Aoki, Hirono, \&

72 Tassanakajon, 2009), fosmid library end sequencing (Huang et al., 2011), molecular marker

73 development (Brooker, Benzie, Blair, \& Versini, 2000; A. Tassanakajon, Pongsomboon, Jarayabhand,

74 Klinbunga, \& Boonsaeng, 1998; A. Tassanakajon, Pongsomboon, Rimphanitchayakit, Jarayabhand, \&

75 Boonsaeng, 1997), linkage map construction (Baranski et al., 2014; Wilson et al., 2002), and

76 transcriptomic analysis (Karoonuthaisiri et al., 2009; Leelatanawit, Uawisetwathana, Klinbunga, \&

77 Karoonuthaisiri, 2011; Lehnert, Wilson, Byrne, \& Moore, 1999; Pootakham, Uengwetwanit, 
78 Sonthirod, Sittikankaew, \& Karoonuthaisiri, 2020; Sittikankaew et al., 2020; Supungul et al., 2002;

79 Anchalee Tassanakajon et al., 2006; Tong, Lehnert, Byrne, Kwan, \& Chu, 2002; Uengwetwanit et al.,

80 2018; Wongsurawat et al., 2010) were explored with limited success. Previous attempts to obtain

81 genome sequences in the black tiger shrimp relied primarily on short-read sequencing platforms due to

82 cost and technical difficulty in extracting high-quality, high-molecular weight DNA in this species

83 (Quyen et al., 2020; Yuan et al., 2018). Recently, two draft genomes for P. monodon were made

84 available; however, both versions of the assemblies were highly fragmented, with N50 contig lengths

85 of merely $937 \mathrm{nt}$ (Yuan et al., 2018) and 1,982 nt (Quyen et al., 2020). Even though those resources

86 were useful for the black tiger shrimp genetics, they lacked the contiguity and completeness required

87 for accurate genome annotation and thorough comparative genomics analyses.

88 Here, we combined a long-read sequencing technology and two long-range scaffolding

89 techniques to obtain high-quality, chromosome-scale genome assembly. First, the Pacific Biosciences

90 (PacBio) sequencing platform was employed to generate the preliminary assembly. The PacBio

91 sequencing technology enables contiguous assembly of repetitive regions containing transposable

92 elements and tandem repeats, which are often omitted or highly fragmented in genomic sequences

93 currently available in public databases. We subsequently applied the long-range Chicago (in vitro

94 proximity ligation) and Hi-C (in vivo fixation of chromosomes) scaffolding techniques to further

95 scaffold the preliminary assembly to achieve the first chromosome-scale genome assembly in $P$.

96 monodon. The utility of this dramatically improved assembly in elucidating novel genes involved in

97 growth regulation was demonstrated by the transcriptome analysis of slow-growing and fast-growing

98 black tiger shrimps. Our high-quality, chromosome-scale genome assembly provides a valuable

99 genetic resource for black tiger shrimp breeding programs and future gene expression and comparative

100 genomics studies in this species.

101

\section{Materials and Methods}

\section{Sample collection and DNA extraction}


104 The muscle of a 5-month-old female P. monodon was collected from the Shrimp Genetic

105 Improvement Center (SGIC, Surat Thani, Thailand), immediately frozen in liquid nitrogen and stored 106 at $-80^{\circ} \mathrm{C}$ until use. Frozen muscle was pulverized in liquid nitrogen and genomic DNA was extracted 107 using a Genomic Tip 100/G kit (Qiagen, USA). DNA quantity was measured using a NanoDrop ND1088000 spectrophotometer and a Qubit dsDNA BR Assay kit (Invitrogen, USA) using Qubit

109 fluorometer. The DNA quality and integrity were visualized under pulsed-field gel electrophoresis at 11080 volts for $9 \mathrm{~h}$ in $0.5 \mathrm{x}$ KBB buffer (51 mM Tris, $28 \mathrm{mM}$ TASP, $0.08 \mathrm{mM}$ EDTA, pH 8.7) (Sage

111 Science, USA) containing SYBR Safe DNA gel staining (Invitrogen, USA).

\section{PacBio library preparation and sequencing}

114 Whole genome sequencing was performed using long read PacBio RS II and SEQUEL (Pacific

115 Biosciences, Menlo Park, USA, outsourced to NovogenAIT, Singapore). The15-kb and 20-kb

116 SMRTbell libraries were constructed for the PacBio RSII and SEQUEL systems, respectively. For

117 short read sequencing, the paired-end library with 150 bp was prepared and sequenced Illumina HiSeq

1182000 (Illumina, San Diego, USA) that was outsourced to Novogene, USA

\section{Illumina library preparation and sequencing}

The short paired-end library (2x150 bp) was sequenced on the Illumina instrument at

122 Novogene (USA). These illumine reads (133X coverage) were used to correct error reads of de novo

123 assembly.

\section{Chicago library preparation and sequencing}

126 A Chicago library was prepared as described previously (Putnam et al., 2016). Approximately,

127 500ng of high molecular weight genomicDNA (mean fragment length $=60 \mathrm{kbp}$ ) was reconstituted into

128 chromatin in vitro and fixed with formaldehyde. Fixed chromatin was digested with DpnII, the 5'

129 overhangs filled in with biotinylated nucleotides, and then free blunt ends were ligated. After ligation, 
130 crosslinks were reversed to remove protein from DNA. Purified DNA was treated to remove biotin

131 that was not internal to ligated fragments. The DNA was then sheared to 350 bp mean fragment size

132 and sequencing libraries were generated using NEBNext Ultra enzymes and Illumina-compatible

133 adapters. Biotin-containing fragments were isolated using streptavidin beads before PCR enrichment

134 of each library. The libraries were sequenced on an Illumina HiSeq X to produce 444 million 2x150 bp

135 paired end reads, which provided $51.89 \mathrm{x}$ physical coverage of the genome (1-100 kb pairs).

136

\section{Dovetail Hi-C library preparation and sequencing}

138 A Dovetail Hi-C library was prepared in a similar way as described previously (Lieberman-

139 Aiden et al., 2009). For each library, chromatin was fixed in place with formaldehyde in the nucleus,

140 and then extracted fixed chromatin was digested with DpnII, the 5' overhangs filled in with

141 biotinylated nucleotides, and then free blunt ends were ligated. After ligation, crosslinks were reversed

142 to remove protein from DNA. The purified DNA were then processed as similar as aforementioned in

143 Chicago library preparation. The libraries were sequenced on an Illumina HiSeq X to produce 430

144 million $2 \times 150$ bp paired end reads, which provided $24,424.63 \mathrm{X}$ physical coverage of the genome (10-

$14510,000 \mathrm{~kb}$ pairs).

146

147 Genome assembly and scaffolding

148 Three types of sequencing reads from Illumina, PacBio, and Dovetail (Chicago and Hi-C reads)

149 were used to construct $P$. monodon genome. PacBio sequence data was used for de novo assembly and

150 Illumina sequence data was subsequently used for polishing to obtain high quality contigs. Chicago

151 and Hi-C reads were used for scaffolding on the high quality contigs to obtain high quality $P$.

152 monodon draft genome. In brief, high quality Illumina reads were prepared using TrimGalore

153 (https://github.com/FelixKrueger/TrimGalore) based on the following criteria: (i) no "N" base, (ii)

154 trimming of adaptor sequences and low quality bases $(\mathrm{Q}<20)$, (iii) no trimmed reads $<100 \mathrm{bp}$. To

155 avoid mis-assembly due to repetitive sequences (Tørresen et al., 2019), PacBio SEQUEL subreads 
156 with repetitive sequences comprised over $85 \%$ of total sequences were filtered out. The GC content

157 criteria $(<25 \%$ and $>85 \%)$ was applied for filtering low complexity DNA sequences before assembly.

158 Noted that GC content of crustacean are 35-41\% (Gao et al., 2017; Yu et al., 2015; Zhao et al., 2012).

159 Moreover, the reads matched mitochondria sequence (NC_002184.1) were excluded from nucleus

160 sequences and processed separately (Supplemental method). The reads $\geq 5,000$ bp were assembled

161 using WTDBG2 (Hu et al., 2019). Illumina reads were then aligned to the assembled contigs by

162 minimap2 (Li, 2018) and subjected for polishing using wtpoa-cns mode in WTDBG2 (Hu et al.,

163 2019).

164 Scaffolding of the genome assemblies was performed using HiRise, a software pipeline

165 designed specifically for using proximity ligation data to scaffold genome assemblies (Putnam et al.,

166 2016). The input de novo assembly, shotgun reads, Chicago library reads, and Dovetail Hi-C library

167 reads were used as input data for HiRise. An iterative analysis was conducted. First, shotgun and

168 Chicago library sequences were aligned to the draft input assembly using a modified SNAP read

169 mapper (http://snap.cs.berkeley.edu). The separations of Chicago read pairs mapped within draft

170 scaffolds were analyzed by HiRise to produce a likelihood model for genomic distance between read

171 pairs, and the model was used to identify and break putative misjoins, to score prospective joins, and

172 make joins above a threshold. After aligning and scaffolding Chicago data, Dovetail Hi-C library

173 sequences were aligned and scaffolded following the same method. After scaffolding, shotgun

174 sequences were used to close gaps between contigs.

175 The P. monodon genome sequence was aligned to the Pacific white shrimp L. vannamei (Zhang

176 et al., 2019) using Mugsy v1.2.3 (Angiuoli \& Salzberg, 2011). Alignments with a length $<\square 1 \mathrm{~kb}$ were

177 filtered out. The output alignments between genomes were visualized using Circos v0.69 $\square 9$

178 (Krzywinski et al., 2009).

\section{Repetitive element analysis}


181 Species-specific repeat library was generated using RepeatModeler2 (Flynn et al., 2019) prior

182 masking with RepeatMasker (Smit, Hubley, \& Green, 2013-2015). Annotation of repeats was aligned

183 to Repbase using RMBlast. All processes were performed with default parameters.

\section{RNA isolation, PacBio Iso-Seq library preparation sequencing and analysis}

186 Total RNA was extracted from gill, heart, hemocyte, hepatopancreas, intestine, ovary, testis,

187 pleopods, stomach and thoracic ganglia of 4-month-old shrimps using TRI REAGENT according to

188 the manufacturer's instruction (Molecular Research Center, USA). Contaminated genomic DNA was

189 removed by treatment with DNase I at $0.5 \mathrm{U} / \mu \mathrm{g}$ total RNA at $37^{\circ} \mathrm{C}$ for $30 \mathrm{~min}$. The DNA-free RNA

190 was subjected for sequencing analysis using PacBio Iso-Seq SEQUEL platform (Pacific Biosciences,

191 Menlo Park, USA, outsourced to NovogenAIT, Singapore) and ONT platform. Sequences obtained

192 from Iso-Seq were prepared as described in Pootakham study (Pootakham et al., 2020).

193

\section{Gene prediction and annotation}

195 Gene prediction and protein-coding sequence identification were performed using a

196 combination of transcriptome-based prediction, homology-based prediction and ab initio prediction

197 methods using EvidenceModeler (Haas et al., 2008) to generate consensus gene prediction for training

198 species-specific parameter in AUGUSTUS (Stanke, Diekhans, Baertsch, \& Haussler, 2008). To locate

199 intron and exon regions, transcriptome-based prediction methods combined information from PacBio

200 Iso-seq and other available P. monodon transcriptome databases (PRJNA4214000, SRR1648423,

201 SRR1648424, SRR2191764, SRR2643301, SRR2643302, SRR2643304, SRR2643305) (Supporting

202 Information Table S1) to align against the genome sequence. For short-read transcripts, STAR (Dobin

203 et al., 2013) was employed to align against the genome before spliced read information was generated

204 according to a previously published protocol (Hoff \& Stanke, 2019) using bam2 wig script in

205 AUGUSTUS. Iso-Seq raw reads containing both 5' and 3' adapters (derived from full-length

206 transcripts) were identified, and the adapters and poly(A) sequences were trimmed. Cleaned consensus 
207 reads were then mapped using Genomic Mapping and Alignment Program (GMAP) (Wu \& Watanabe,

208 2005). Expressed sequence tags (Anchalee Tassanakajon et al., 2006) were aligned to the genome

209 using BLAT (Kent, 2002) and converted to potential gene structures using blat2hints script of

210 AUgustus.

211 Protein sequences of $P$. monodon were mapped against proteins from closely related organisms

212 including H. azteca, and L. vannamei, using Exonerate version 2.2.0 (Slater \& Birney, 2005). All gene

213 models derived from these three methods were integrated by EvidenceModeler into a high confident

214 nonredundant gene set, which was used to set species-specific parameters. Finally, AUGUSTUS was

215 used to predict genes in the genome based on the extrinsic evidence. Functional annotations of the

216 obtained gene set were conducted using Semantic Annotation Platform (SAPP) using the InterProScan

217 module (P. Jones et al., 2014; Koehorst et al., 2018) and Blast2GO (Götz et al., 2008).

218

Phylogenetic analysis

Mitochondria protein-coding genes were used to construct a molecular phylogenetic analysis using MEGA7 software (Kumar, Stecher, \& Tamura, 2016). The amino acid sequences of 22 species,

222 which were Anopheles quadrimaculatus, Anopheles gambiae, Apis mellifera,, Artemia franciscana,

223 Ceratitis capitata, Daphnia pulex, Drosophila yakuba, Drosophila melanogaster, Euthynnus affinis,

224 Halocaridina rubra, Hyalella azteca, Ixodes hexagonus, , L. vannamei, Ligia oceanica, Locusta

225 migratoria, Macrobrachium rosenbergii, Panulirus japonicus, Parhyale hawaiensis, P. monodon,

226 Portunus trituberculatus, Rhipicephalus sanguineus, and Tigriopus japonicus were used for the

227 analysis. The evolutionary history was inferred by using the maximum likelihood method based on the

228 JTT matrix-based model (D. T. Jones, Taylor, \& Thornton, 1992). The tree with the highest log

229 likelihood (-84614.94) is shown. An initial tree for heuristic search was obtained automatically by

230 applying Neighbor-Join and BioNJ algorithms to a matrix of pairwise distances estimated using a JTT

231 model, and then selecting the topology with superior log likelihood value. 
232 Pan-core protein families were constructed for P. monodon with the available crustacean

233 genome E. affanis, D. pulex, P. hawaiensis, L. vannamei and H. azteca. The orthologous genes protein

234 family were identified by clustering together using MMseq2 (Steinegger \& Söding, 2017) with

235 standard parameters of 0.5 identity and 0.5 coverage. The pan-core protein family matrix was used to

236 construct a pan-core protein family tree follow the method presented by Snipen et al (Snipen \&

237 Ussery, 2010). The comparison of common and specific protein family among the crustacean genomes

238 was present in the upset plot (Lex, Gehlenborg, Strobelt, Vuillemot, \& Pfister, 2014). The pan-core

239 protein family tree was used to construct an ultrametric tree using ape v5.3 library in R v3.6.1 (Paradis

240 \& Schliep, 2018; R Core Team, 2019). The ultrametric tree and matrix of protein families were

241 subjected to CAFE v4.1 (Bie, Cristianini, Demuth, \& Hahn, 2006) to study the evolution of gene

242 families in P. monodon. The resulting families were further filtered to exclude families with large

243 ranges in size (> 25) across the tree, leaving 102619 families for expansion and contraction analysis.

244 CAFE was used to obtain a maximum likelihood estimate of a global birth and death rate parameter $\lambda$

245 (lambda; the rate of gain/loss per gene per million years) across the species tree $(\lambda=0.27)$. The size of

246 families was estimated at each ancestral node and then used to obtain a family-wise p-value to indicate

247 non-random expansion or contraction for each family and across all branches of the tree.

Transcriptome sequencing and analyses of the fast-growing and slow-growing groups

The feeding trial was carried out at SGIC. Black tiger shrimps were cultured in a pond size 800 $\mathrm{m}^{2}\left(25\right.$ shrimps $\left./ \mathrm{m}^{2}\right)$. Five-month-old female shrimps $(N=140)$ were randomly selected for body weight measurement (Supporting Information Figure S1). Two experimental groups were separated base on the lowest and highest shrimp weight, called the "slow-growing" group and the "fast-growing" group, 
258 individual frozen hepatopancreas tissues were pulverized in liquid nitrogen and subjected to TRI-

259 REAGENT extraction method and DNase treatment as previously described.

260 To obtain short-read RNA sequences, 30 libraries ( $\mathrm{n}=15$ for each group) were constructed using

261 the HiSeq Library Preparation kit (Illumina, San Diego, USA) and sequenced using Illumina

262 NovaSeq ${ }^{\mathrm{TM}}$. Illumina sequencing using Novaseq 6000 instrument with 150 pair-end reads was

263 performed at Omics Drive, Singapore. The raw reads were quality-filtered $(\mathrm{Q}<20,>50 \mathrm{bp})$ and adapter-

264 trimmed using TrimGalore (https://github.com/FelixKrueger/TrimGalore). The reads were mapped to

265 the genome using STAR (Dobin et al., 2013). The HTSeq-count(Anders, Pyl, \& Huber, 2015) and

266 DESeq2 (Love, Huber, \& Anders, 2014) provide a test for differential expression using negative

267 binomial generalized linear models, will operate to identify significant differently expressed genes

268 (DEGs) between fast- and slow-growing shrimp. DEGs were identified when their expression level

269 differences $>2.0$ change with Bonferroni adjusted p-value of $<0.05$. The DEGs which have read

270 count per million < 1 were discarded. Functional pathway analysis was carried using EggNOG

271 (Huerta-Cepas et al., 2019) and KEGG mapper (Kanehisa, Sato, Kawashima, Furumichi, \& Tanabe,

272 2016). To determine whether the differentially expressed genes may be newly identified genes in $P$.

273 monodon, the sequences were aligned on available $P$. monodon protein and mRNA sequences in NCBI

274 database using BLAST (E-value $\leq 10^{-3}$ ). The sequences that had no match to any publicly available $P$.

275 monodon sequences but could be annotated were considered newly identified genes.

276 To evaluate differentially expressed genes, 15 genes (10 DEGs that had higher levels of

277 expression in the fast-growing shrimp and 5 DEGs that had higher levels of expression in the slow-

278 growing shrimp) were selected for real-time PCR analysis. Eight hepatopancreas per group (fast-

279 growing and slow-glowing) were used for validation. Total RNA (1.5 $\mu \mathrm{g})$ was reverse transcribed into

280 cDNA using an ImPromII ${ }^{\mathrm{TM}}$ Reverse Transcriptase System kit (Promega, USA.) according to the

281 manufacturer's recommendation. Each $20 \mu \mathrm{L}$ qPCR reaction included $200 \mathrm{ng}$ cDNA, $0.2 \mu \mathrm{M}$ of each

282 primer, and SsoAdvanced ${ }^{\mathrm{TM}}$ Universal SYBR ${ }^{\circledR}$ Green supermix (BioRad, USA.) according to the

283 company's instruction. The thermal cycling parameters were $95^{\circ} \mathrm{C}$ for $2 \min 30 \mathrm{~s}$, followed by 40 
284 cycles of $95^{\circ} \mathrm{C}$ for $15 \mathrm{~s}, 58^{\circ} \mathrm{C}$ for $20 \mathrm{~s}$ and $72^{\circ} \mathrm{C}$ for $30 \mathrm{~s}$. The melting curve analysis was performed 285 from $65^{\circ} \mathrm{C}$ to $95^{\circ} \mathrm{C}$ with a continuous fluorescent reading with a $0.5^{\circ} \mathrm{C}$ increment. The threshold cycle 286 (Ct) was analyzed using BioRad CFX Manager 2.1 software (BioRad, USA).

\section{Results}

\section{Genome sequencing, assembly and annotation} genome from PacBio long-read data. A total of 13,157,113 raw reads (178.94 Gb) representing 69.08X coverage based on the estimated genome size of $2.59 \mathrm{~Gb}$ obtained from a previous report using the flow cytometry method (Swathi, Shekhar, Katneni, \& Vijayan, 2018). De novo assembly of PacBio sequences yielded a preliminary assembly of $2.39 \mathrm{~Gb}$ (70,380 contigs) with a contig N50 of $79 \mathrm{~kb}$ and a L50 of 6,786 contigs (Table 1). The draft genome was further assembled using the long-range length (hereafter referred to as chromosomes, numbered according to size; Figure 1A), corresponding to the haploid chromosome number in P. monodon $(1 n=44,2 n=88)$. The 44 chromosomes covered $1,986,035,066$ bases or $82.9 \%$ of the $2.39-\mathrm{Gb}$ assembly.

303 Illumina sequencing from this work and the previous report (Yuan et al., 2018) to the assembled

304 genome and found that approximately 93\% of the DNA short reads could be mapped on the here 305 reported de novo assembly. We also aligned the publicly available RNA-seq reads (Huerlimann et al., 306 2018) and Iso-seq reads (Pootakham et al., 2020) to the assembly, and $90.22 \%$ and $98.77 \%$ of the 307 RNA-seq and Iso-seq reads were mapped to the assembly, respectively. To further assess the 308 completeness of the genome assembly, we checked the gene content with the BUSCO software using 309 the Eukaryota (odb9) database (Simão, Waterhouse, Ioannidis, Kriventseva, \& Zdobnov, 2015). Our 
310 gene prediction contained $94.72 \%$ of the highly conserved orthologs ( $85.15 \%$ complete, $9.57 \%$ partial,

$3115.28 \%$ missing) in the eukaryotic lineage. High mapped rates, comparable to the numbers observed in

312 L. vannamei, and a high percentage of identified highly conserved orthologs provided the evidence for

313 a high-quality assembly obtained for the black tiger shrimp genome.

314 The chromosome-level contiguity achieved in our assembly enabled a syntenic analysis between

315 P. monodon and L. vannamei. Portions of conserved syntenic genes between the black tiger shrimp

316 and Pacific white shrimp are illustrated in Figure 1B and 1C. The degree of macrosynteny observed

317 between these two species was consistent with the result from phylogenetic analysis showing that they

318 are very closely related and appear to have evolved from a common ancestor (Figure 1D). The

319 distribution of paralogous gene pairs revealed one-to-one synteny across 15 chromosomes/linkage

320 groups and one-to-two synteny between P. monodon chromosomes 4 and 30 and L. vannamei

321 chromosomes 14, 15, 21 and 22 (Figure 1B).

322 A combination of ab initio prediction, homology-based search and transcript evidence obtained

323 from both Iso-seq and RNA-seq data was used for gene prediction. The genome annotations of $P$.

324 monodon contained 31,640 predicted gene models, of which 30,038 were protein-coding genes (Table

325 1, Supporting Information Table S3). The number of protein-coding genes in P. monodon was slightly

326 higher than those reported in L. vannamei $(25,596)$ (Zhang et al., 2019) and in a freshwater epibenthic

327 amphipod Hyalella azteca (19,936) (Poynton et al. 2018). Of 30,038 protein-coding genes, 25,569

$328(85.02 \%)$ had evidence support from our RNA-seq and Iso-seq data.

329 In addition to the nuclear genome, a complete mitochondrial genome assembly was obtained,

330 enabling phylogenic analysis to reveal relationship with other arthropods. The assembled

331 mitochondrial genome had a size of 15,974 bp and $29.09 \%$ GC content (Supporting Information

332 Figure S2). The phylogenetic analysis of 20 arthropods based on 13 concatenated mitochondrial

333 protein-coding genes showed that $P$. monodon and other Decapoda were more closely related to the

334 insects than other crustaceans in the same class (Malocostraca) such as H. azteca and Parhyale

335 hawaiensis (Amphipoda), consistent with a previous study (Wilson, Cahill, Ballment, \& Benzie, 2000) 
336 (Figure 1D). The phylogenic tree also showed that decapods were distantly related to crustaceans in

337 Branchiopoda class such as Artemia franciscana and Daphnia pulex.

338

\section{Comparative analyses between $P$. monodon and other crustacean genomes}

340 Comparative analyses of $P$. monodon genome with publicly available crustacean genomes

341 (Calanoida (Eurytemora affinis) (Eyun et al., 2017), Cladocera (D. pulex) (Colbourne et al., 2011) and

342 Amphipoda (P. hawaiensis (Kao et al., 2016), L. vannamei (Zhang et al., 2019) and H. azteca

343 (Poynton et al., 2018)) were performed to investigate genome evolution within the crustacean group.

344 Based on orthologous clustering by MMseq2 software (Steinegger \& Söding, 2017), we identified

345102,632 pan protein families of the crustacean with the core protein family of 583 (Figure 2A). The

346 dendrogram analysis of protein families showed a distinct group of the Amphipoda. As expected of the

347 closely related species, P. monodon and L. vannamei shared 5,487 common protein families that were

348 higher than those between P. monodon and other species. Nevertheless, both species also had around

34911,000 species-specific protein families (Figure 2A). The numbers of expanded and contracted gene

350 numbers at each divergence event were estimated using CAFÉ software (Bie et al., 2006).

351 Considerable gene family expansions occurred on the P. monodon branch that might have experienced 352 additional expansions after its divergence from $H$. azteca.

\section{Repetitive elements in $P$. monodon genome}

355 The total repeat content in the black tiger shrimp genome assembly was $62.5 \%$ (Figure $2 \mathrm{~B}$ ). We

356 identified repeats comprising 572.87 Mb simple repeats (23.93\%), 276.64 Mb long interspersed

357 nuclear elements (LINEs, 11.55\%), 93.21 Mb long terminal repeats (LTRs, 3.89\%), 75.96 Mb DNA

358 elements (3.17\%), 59.09 Mb low complexity repeats (2.47\%), 49.49 Mb short interspersed nuclear

359 elements (SINEs, 2.07\%), 2.18 Mb small RNA (0.09\%), and 368.13 Mb of unclassified repeat

360 elements (15.37\%). 
366 (Yuan et al., 2018).

\section{Identification of novel growth-associated genes}

Transcriptome analysis is a useful approach to identify genes that are differentially expressed among individuals with various traits of interest. Without a high-quality reference genome, gene

371 expression studies have to rely on a de novo transcriptome assembly, which often contains a large 372 number of fragmented transcript sequences with no annotation or in some cases incorrect annotations. 373 To evaluate the utility of this high-quality genome assembly in transcriptomic analysis, 374 transcriptomics of hepatopancreas from the fast- and slow-growing shrimps were compared with and 375 without the genome assembly as a reference.

376 Comparison of the overall results from the genome assembly as a reference and from de novo 377 transcriptome assembly revealed a significant improvement. With the genome assembly, a lower 378 number of predicted genes with a higher N50 value was obtained (31,640 genes with N50 of 1,743 nt 379 from the genome assembly vs 340,240 genes with N50 of $776 \mathrm{nt}$ from the de novo transcriptome 380 assembly). Moreover, a higher annotation rate (95.01\%) was obtained with the genome assembly as a 381 reference than that from the de novo transcriptome assembly $(26.36 \%)$.

382 We compared the differential gene expression profile in hepatopancreas of the fast-growing 383 shrimp and the slow-growing shrimp. This analysis identified 383 genes exhibiting higher levels of 384 expression in the fast-growing shrimp and 95 genes exhibiting higher levels in the slow-growing 385 shrimp ( $\log _{2}$ fold-change>1 and $p$-value <0.05) (Supporting Information Table S4). The fast-growing 386 shrimp grew at a faster rate and had twice the weight of the slow-growing shrimp under the same 
387 rearing condition at 5 months old. To further access gene interaction networks, KEGG pathway 388 mapping was employed on the DEGs and revealed 159 pathways with an average of two genes 389 associated in each pathway (Supporting Information Table S5). The functions of DEGs were classified 390 by Clusters of Orthologous Groups (COGs) annotation into 23 categories (Supporting Information 391 Figure S3). The top five highly enriched metabolic processes were carbohydrate/lipid/amino acid 392 transport and metabolism, secondary metabolites biosynthesis, transport and catabolism, and inorganic 393 ion transport and metabolism (Figure 3A). For instance, DEGs involved in carbohydrate metabolism 394 were amylase (amy), fructose-bisphosphate aldolase (aldo), glyceraldehyde 3-phosphate 395 dehydrogenase (gapdh), and insulin-like growth factor-1 receptor (insr). Genes involved in lipid 396 metabolism were nose resistant to fluoxetine (nrf), lipase (lip), glycerol-3-phosphate acyltransferase 397 (gpat3), acyl-CoA delta desaturase (scd), long-chain-fatty-acid--CoA ligase (acsl1) and elongation of 398 very-long-chain fatty acids protein 4-like (elovl4). Of all DEGs, 67 annotated genes could not be 399 matched with any P. monodon sequences in the publicly available genomic/transcriptomic databases 400 (Supporting Information Table S4). These newly identified genes involved in nutrient metabolic 401 processes (Figure 3A shown in blue). We further investigated pathways that have not been reported to 402 be involved in shrimp growth and found that PI3K-Akt signaling pathway has the highest number of 403 novel genes (Figure 3B). Four DEGs in PI3K-Akt signaling pathway were integrin beta isoform 1 404 (itgb1), integrin alpha-4 like (itga4), serine/threonine-protein kinase N isoform 1 (pkn1) and insr. Of 405 these, the last three were newly identified genes.

406 To verify the transcriptome analysis, 15 DEGs with functions related to nutrient metabolism or 407 immune system were selected for quantitative real-time PCR (qPCR). The qPCR results agreed with 408 those obtained from RNA-seq with a correlation coefficient of 0.96 (Supporting Information Figure 409 S4).

\section{Discussion}


412 In this study, we present a whole genome sequence of $P$. monodon assembled from PacBio

413 long-read data and scaffolded using long-range Chicago and $\mathrm{Hi}-\mathrm{C}$ techniques. Our chromosome-scale

414 assembly has shown tremendous improvement in contiguity and completeness compared to the

415 previously reported P. monodon genomes (Quyen et al., 2020; Yuan et al., 2018). Based on a

416 benchmark (10Mb) (Reference standard for genome biology, 2018), the assembly presented here is

417 considered a high-quality reference genome as it has the N50 scaffold length of $44.86 \mathrm{Mb}$. It is also

418 one of the highest quality crustacean genomes currently available. Of the 45 crustacean genome

419 sequences listed in the NCBI genome database, only three species (Tigriopus japonicus, T.

420 californicus and Daphnia magna) have their genomes assembled at a chromosome level.

421 The present genome provides an invaluable resource for shrimp research. The availability of the

422 chromosome-scale assembly allowed us to examine the syntenic relationship between the two

423 economically important penaeid shrimp species: the black tiger shrimp and the Pacific white shrimp

424 genomes. The synteny analysis revealed a one-to-one relationship between $P$. monodon and $L$.

425 vannamei chromosomes, suggesting that certain chromosomes derived from the common ancestor of

426 P. monodon and L. vannamei were fragmented into two smaller chromosomes in L. vannamei (for

427 example, P. monodon chromosome 4 displayed synteny to L. vannamei pseudochromosomes 14 and

428 15; Figure 1B) but remained as single chromosomes in P. monodon.

429 Obtaining contiguous long-read-based genome of shrimp has been hampered by limitation

430 related to short-read sequencing technology. PacBio sequencing reads allow the assembler to obtain a

431 contiguous assembly that spans repeat regions containing transposable elements and tandem repeats.

432 We found that P. monodon has the highest repeat abundance (62.5\%) when compared to five available

433 genome sequences of crustacean species: L. vannamei (53.9\%), P. hawaiensis (44.7\%), E. affinis

434 (40.2\%), H. azteca (24.3\%) and D. pulex (22.0\%). Moreover, the percentage of repeat elements

435 observed in this assembly was substantially higher than reported in previous studies on $P$. monodon

436 fosmid (51.8\%) (Huang et al., 2011) and genome (46.8\%) (Yuan et al., 2018). High repeat contents

437 and long repetitive sequences hindergenome assembly. Ambiguous regions containing mostly 
438 repetitive sequences might be missed or caused errors in assembly using short sequencing reads

439 employed in the previous assembly of the P. monodon genomes (Yuan et al., 2018) and L. vannamei

440 genome (Zhang et al., 2019). Our assembly, on the other hand, utilized the PacBio sequencing

441 platform, which yielded kilobase-sized reads that could be assembled into contigs and scaffolds large

442 enough to span repetitive regions, alleviating the problems often encountered by the use of short-read

443 technologies.

444 The chromosome-scale assembly allows for an in-depth investigation of repeat elements. Even

445 though the biological function of repeats has not been well studied in the shrimp, they might be

446 associated with important functions as reported in other organisms. For example, LINE/I plays a role

447 on transcription in human by co-mobilizing DNA to new locations (Pickeral, Makałowski, Boguski, \&

448 Boeke, 2000). Among diverse repetitive elements, LINE was the major element in both P. monodon

449 and L. vannamei. The most abundant element in P. monodon and L. vannamei were LINE/I and

450 LINE/L2, respectively. Given that the diversity of repeats was deemed to play a role in environmental

451 adaptation of animals (Schrader \& Schmitz, 2019), roles of these repetitive elements in the black tiger

452 shrimp could be further explored to gain a better understanding of shrimp biology.

453 Another advantage of the high-quality reference genome is the reduction in erroneous and

454 fragmented assembled contigs using de novo transcripts. With this high-quality reference genome

455 assembly, we were able to obtain an improved gene set with better contiguity and annotation rate. Of

456 the predicted genes in the genome, $95.01 \%$ of them could be functionally annotated. The results

457 suggested that our genome assembly could serve as a high-quality reference for facilitating functional

458 genomic study in the black tiger shrimp.

459 The chromosome-scale assembly facilitates downstream applications for molecular breeding

460 and gene expression studies in shrimp. Growth is undoubtedly an important factor for profitable

461 shrimp production. However, a daunting challenge in black tiger shrimp farming is its slower growth

462 rate in captivity (Benzie, Kenway, \& Ballment, 2001; Cheng \& Chen, 1990). Domesticated shrimps

463 could not mature well with declining growth rates over generations (Jackson \& Wang, 1998). Albeit 
464 its importance, the knowledge on genes controlling shrimp's growth remained limited partly due to the

465 lack of the genome, thus up-to-now most growth-related genes identified in P. monodon were from $L$.

466 vannamei (Gao et al., 2017; Gao et al., 2015; Santos et al., 2018).

467 The comparison of hepatopancreas transcriptomes of slow-growing shrimp and fast-growing

468 shrimp revealed that DEGs were mainly involved in nutrient metabolism, which was in concordance

469 with the hepatopancreas functions in feed utilization and energy storage. Here, we presented genes

470 related to carbohydrate and lipid metabolisms as they are main nutrients that have been investigated to

471 enhance growth in shrimp (Coelho, Yasumaru, Passos, Gomes, \& Lemos, 2019; González-Félix,

472 Gatlin, Lawrence, \& Perez-Velazquez, 2002; Hu et al., 2019; Olmos, Ochoa, Paniagua-Michel, \&

473 Contreras, 2011). Genes with higher expression levels in the fast-growing group were found to be

474 involved in nutrient metabolism and secondary metabolite biosynthesis. Genes exhibiting higher levels

475 of expression in the fast-growing shrimp were digestive enzymes such as amylase and lipase, in

476 agreement with the prior finding that enhancement of digestive enzyme activities improves growth

477 performance of shrimp activities (Anand et al., 2013; Gómez \& Shen, 2008). As dietary carbohydrates

478 can enhance growth (Sagar et al., 2019), it is not surprising to find carbohydrate metabolism genes

479 such as aldo, gapdh and insr expressed at a higher level in the fast-growing shrimp

480 Similarly, DEGs in lipid metabolism agree with the established knowledge on the importance of

481 fatty acids and lipids to the shrimp growth and immunity (Chen et al., 2015; Duan et al., 2019; Toledo,

482 Silva, Vieira, Mouriño, \& Seiffert, 2016). Many fatty acids such as highly unsaturated fatty acids

483 (HUFA) are indeed essential to marine animals since they are the major component of cell membrane

484 (An et al., 2020). Thus, regulation of the synthesis, digestion and absorption of these fatty acids is a

485 key to shrimp growth. The higher expression of genes involved in unsaturated fatty acid (UFA)

486 biosynthesis such as $s c d$ and acsll in the fast-growing group suggested that these fatty acids might

487 positively associate with growth. The results agree with the previous studies reported that additional of

488 high HUFA such as linoleic (LOA, 18:2n-6), linolenic (LNA, 18:3n-3), eicosapentaenoic acid (EPA,

489 20:5n-3) or docosahexaenoic acid (DHA, 22:6n-3) in diet could promote the growth (Glencross, 
490 Smith, Thomas, \& Williams, 2002a, 2002b). Interestingly, elovl4 that synthesizes very long-chain

491 (>C24) saturated and polyunsaturated fatty acids (Oboh, Navarro, Tocher, \& Monroig, 2017) was

492 expressed at a higher level in the slow-growing shrimp than in the fast-growing one. In gilthead sea

493 bream Sparus aurata, high amount of LNA and long-chain fatty acid adversely affected growth

494 (Turkmen et al., 2019). Considering the above data, it could be implied that balance lipid composition

495 is an important factor controlling growth performance; thus, feed formulation to promote growth

496 should be optimized by considering lipid ratios.

497 Among the DEGs, three new candidate marker genes may potentially be useful for shrimp

498 breeding programs, namely tranferin (trf), $n r f$ and $p k n 1$. Trf is an insulin-like growth factor (IGF) that

499 stimulates both proliferation and differentiation in a cell line (Kiepe, Ciarmatori, Hoeflich, Wolf, \&

500 To $\square$ nshoff, 2005), making it a promising candidate as a growth marker. Nrf, a lipid-carrier protein,

501 has been reported to be essential for embryonic development in Caenorhabditis elegans (Watts \&

502 Browse, 2006). Given the aforementioned importance of lipid for shrimp growth, Nrf might regulate

503 the intake and storage of lipid for the growth. Besides nutrient metabolic pathways, PI3K-Akt pathway

504 presents an interesting pathway for further investigation for its involvement in growth regulation as we

505 found three novel genes ( $p k n 1$, itga4, and insr) in this pathway. PI3K-Akt is a regulatory pathway

506 controlling glucose balance by cross-talking with insulin signaling pathway (Shi \& He, 2016).

507 Although an association between PI3K-Akt and shrimp growth performance has not yet been

508 investigated in P. monodon, there has been a report that PI3K-Akt is linked to growth factors and

509 cellular survival (Choi et al., 2019; Dai, Li, Fu, Qiu, \& Chen, 2020; Fuentes, Valdés, Molina, \&

510 Björnsson, 2013). Here, novel genes associated with PI3K-Akt pathway showed higher expression in

511 the fast-growing shrimp; therefore, it suggested that these genes might have potential roles in

512 promoting growth. Particularly, the distinct expression values of $p k n 1$ between fast- and slow-growing

513 shrimps make it interesting for further study (Supporting Information Figure S4). Pkn1 is found in

514 various tissues with different functions in many animals (Mukai, 2003). For instance, Pkn1 in testis

515 regulates male fertility of the Pacific abalone Haliotis discus hannai, (Kim, Kim, Park, \& Nam, 2019), 
516 whereas it has been linked to the regulation of actin and cytoskeletal network in human (Dong et al.,

517 2000). These newly identified candidate genes might provide better understanding of shrimp growth

518 and facilitate black tiger shrimp genomic breeding programs.

519 In conclusion, we have successfully overcome the technical challenges in obtaining the first

520 high-quality chromosome-scale genome assembly of the economically important $P$. monodon. The

521 availability of this reference genome enables several downstream biological and industrial applications

522 that would otherwise be difficult. This reference genome will benefit not only the P. monodon research

523 community, but also other researchers working on related shrimp and crustacean species. Moreover,

524 the newly identified growth-associated genes might help advance the understanding of growth in the

525 black tiger shrimp and facilitate its genomic breeding programs.

526

\section{Acknowledgements}

528 The authors acknowledge NSTDA Supercomputer Center (ThaiSC) for providing HPC

529 resources that have contributed to the research results reported within this paper. We thank Ms. Somjai

530 Wongtripop, Mr. Kaidtisak kaewlok and Shrimp Genetic Improvement Center (SGIC) members, as

531 well as Ms. Sudtida Phuengwas (BIOTEC) for the shrimp sample collection. This project was funded

532 by National Center for Genetic Engineering and Biotechnology (BIOTEC Platform No. P1651718),

533 TDG Food and Feed program, National Science and Technology Development Agency (No.

534 P1950419) and partially supported by the European Union's Horizon 2020 research and innovation

535 programme under the Marie Skłodowska-Curie grant agreement No 734486 (SAFE-Aqua). Visiting

536 Professor Program 2019 was awarded to Dr. Intawat Nookaew by the National Science and

537 Technology Development Agency, Thailand. We acknowledge Zulema Dominguez (University of

538 Arkansas for Medical Sciences, USA) for technical assistance on comparative genome. We are

539 grateful to Prof. Morakot Tanticharoen (NSTDA), Dr. Kanyawim Kirtikara (BIOTEC), Dr. Wonnop

540 Visessanguan (BIOTEC) and Assoc Prof. Skorn Koonawootrittriron (Kasetsart University, Thailand)

541 for their mentorship on the shrimp research programs. 


\section{References}

An, W., He, H., Dong, X., Tan, B., Yang, Q., Chi, S., . . Yang, Y. (2020). Regulation of growth, fatty acid profiles, hematological characteristics and hepatopancreatic histology by different dietary n-3 highly unsaturated fatty acids levels in the first stages of juvenile Pacific white shrimp (Litopenaeus vannamei). Aquaculture Reports, 17, 100321. doi:10.1016/j.aqrep.2020.100321

Anand, P. S. S., Kohli, M. P. S., Roy, S. D., Sundaray, J. K., Kumar, S., Sinha, A., . . . Sukham, M. k. (2013). Effect of dietary supplementation of periphyton on growth performance and digestive enzyme activities in Penaeus monodon. Aquaculture, 392-395, 59-68. doi:10.1016/j.aquaculture.2013.01.029

Anders, S., Pyl, P. T., \& Huber, W. (2015). HTSeq--a Python framework to work with highthroughput sequencing data. Bioinformatics, 31(2), 166-169. doi:10.1093/bioinformatics/btu638

Anderson, J. L. (2019, 4 November 2019). GOAL 2019: Global shrimp production review. Retrieved from https://www.aquaculturealliance.org/advocate/goal-2019-global-shrimp-productionreview/

Angiuoli, S. V., \& Salzberg, S. L. (2011). Mugsy: fast multiple alignment of closely related whole genomes. Bioinformatics, 27(3), 334-342. doi:10.1093/bioinformatics/btq665

Baranski, M., Gopikrishna, G., Robinson, N. A., Katneni, V. K., Shekhar, M. S., Shanmugakarthik, J., ... Ponniah, A. G. (2014). The development of a high density linkage map for black tiger shrimp (Penaeus monodon) based on cSNPs. PLoS One, 9(1), e85413. doi:10.1371/journal.pone.0085413

Benzie, J. A. H., Kenway, M., \& Ballment, E. (2001). Growth of Penaeus monodon x Penaeus esculentus tiger prawn hybrids relative to the parental species. Aquaculture, 193(3-4), 227237. doi:10.1016/s0044-8486(00)00487-7

Bie, T. D., Cristianini, N., Demuth, J. P., \& Hahn, M. W. (2006). CAFE: a computational tool for the study of gene family evolution. Bioinformatics, 22(10), 1269-1271. doi:10.1093/bioinformatics/bt1097

Brooker, A. L., Benzie, J. A. H., Blair, D., \& Versini, J. J. (2000). Population structure of the giant tiger prawn Penaeus monodon in Australian waters, determined using microsatellite markers. Marine Biology, 136(1), 149-157. doi:10.1007/s002270050017

Chen, K., Li, E., Xu, C., Wang, X., Lin, H., Qin, J. G., \& Chen, L. (2015). Evaluation of different lipid sources in diet of pacific white shrimp Litopenaeus vannamei at low salinity. Aquaculture Reports, 2, 163-168. doi:10.1016/j.aqrep.2015.10.003

Cheng, C. S., \& Chen, L.-c. (1990). Growth characteristics and relationships among body length, body weight and tail weight of Penaeus monodon from a culture environment in Taiwan. Aquaculture, 91(3-4), 253-263. doi:10.1016/0044-8486(90)90192-p

Choi, E., Kikuchi, S., Gao, H., Brodzik, K., Nassour, I., Yopp, A., . . Y Yu, H. (2019). Mitotic regulators and the SHP2-MAPK pathway promote IR endocytosis and feedback regulation of insulin signaling. Nat Commun, 10(1), 1473. doi:10.1038/s41467-019-09318-3

Coelho, R. T. I., Yasumaru, F. A., Passos, M. J. A. C. R., Gomes, V., \& Lemos, D. (2019). Energy budgets for juvenile Pacific whiteleg shrimp Litopenaeus vannamei fed different diets. Brazilian Journal of Oceanography, 67, e19243. doi:10.1590/s1679-87592019024306701

Colbourne, J. K., Pfrender, M. E., Gilbert, D., Thomas, W. K., Tucker, A., Oakley, T. H., . . Boore, J. L. (2011). The ecoresponsive genome of Daphnia pulex. Science, 331(6017), 555-561. doi:10.1126/science.1197761

Dai, M., Li, S., Fu, C., Qiu, H., \& Chen, N. (2020). The potential role of marine protein hydrolyzates in elevating nutritive values of diets for largemouth bass, Micropterus salmoides. Frontiers in Marine Science, 7, 197 doi:10.3389/fmars.2020.00197 
Dobin, A., Davis, C. A., Schlesinger, F., Drenkow, J., Zaleski, C., Jha, S., . . Gingeras, T. R. (2013). STAR: ultrafast universal RNA-seq aligner. Bioinformatics, 29(1), 15-21. doi:10.1093/bioinformatics/bts635

Dong, L. Q., Landa, L. R., Wick, M. J., Zhu, L., Mukai, H., Ono, Y., \& Liu, F. (2000). Phosphorylation of protein kinase $\mathrm{N}$ by phosphoinositide-dependent protein kinase-1 mediates insulin signals to the actin cytoskeleton. Proceedings of the National Academy of Sciences of the United States of America, 97(10), 5089-5094. doi:10.1073/pnas.090491897

Duan, Y., Wang, Y., Liu, Q., Dong, H., Li, H., Xiong, D., \& Zhang, J. (2019). Changes in the intestine microbial, digestion and immunity of Litopenaeus vannamei in response to dietary resistant starch. Scientific Reports, 9(1), 6464. doi:10.1038/s41598-019-42939-8

Eyun, S.-I., Soh, H. Y., Posavi, M., Munro, J. B., Hughes, D. S. T., Murali, S. C., . . Lee, C. E. (2017). Evolutionary history of chemosensory-related gene families across the Arthropoda. Molecular Biology and Evolution, 34(8), 1838-1862. doi:10.1093/molbev/msx147

FAO. (2018). The State of World Fisheries and Aquaculture 2018 - Meeting the sustainable development goals [Press release]

Flynn, J. M., Hubley, R., Goubert, C., Rosen, J., Clark, A. G., Feschotte, C., \& Smit, A. F. (2019). RepeatModeler2: automated genomic discovery of transposable element families. bioRxiv, 856591. doi:10.1101/856591

Fuentes, E. N., Valdés, J. A., Molina, A., \& Björnsson, B. T. (2013). Regulation of skeletal muscle growth in fish by the growth hormone - Insulin-like growth factor system. General and Comparative Endocrinology, 192, 136-148. doi:10.1016/j.ygcen.2013.06.009

Gao, Y., Wei, J., Yuan, J., Zhang, X., Li, F., \& Xiang, J. (2017). Transcriptome analysis on the exoskeleton formation in early developmetal stages and reconstruction scenario in growthmoulting in Litopenaeus vannamei. Scientific Reports, 7(1), 1098. doi:10.1038/s41598-01701220-6

Gao, Y., Zhang, X., Wei, J., Sun, X., Yuan, J., Li, F., \& Xiang, J. (2015). Whole transcriptome analysis provides insights into molecular mechanisms for molting in Litopenaeus vannamei. PLoS One, 10(12), e0144350. doi:10.1371/journal.pone.0144350

Glencross, B. D., Smith, D. M., Thomas, M. R., \& Williams, K. C. (2002a). The effect of dietary n-3 and n- 6 fatty acid balance on the growth of the prawn Penaeus monodon. Aquaculture Nutrition, 8(1), 43-51. doi:10.1046/j.1365-2095.2002.00188.x

Glencross, B. D., Smith, D. M., Thomas, M. R., \& Williams, K. C. (2002b). Optimising the essential fatty acids in the diet for weight gain of the prawn, Penaeus monodon. Aquaculture, 204(1-2), 85-99. doi:10.1016/s0044-8486(01)00644-5

Gómez, R. G. D., \& Shen, M. A. (2008). Influence of probiotics on the growth and digestive enzyme activity of white Pacific shrimp (Litopenaeus vannamei). Journal of Ocean University of China, 7(2), 215-218. doi:10.1007/s11802-008-0215-x

González-Félix, M. L., Gatlin, D. M., Lawrence, A. L., \& Perez-Velazquez, M. (2002). Effect of dietary phospholipid on essential fatty acid requirements and tissue lipid composition of Litopenaeus vannamei juveniles. Aquaculture, 207(1-2), 151-167. doi:10.1016/s00448486(01)00797-9

Götz, S., García-Gómez, J. M., Terol, J., Williams, T. D., Nagaraj, S. H., Nueda, M. J., . . Conesa, A. (2008). High-throughput functional annotation and data mining with the Blast2GO suite. Nucleic Acids Research, 36(10), 3420-3435. doi:10.1093/nar/gkn176

Guppy, J. L., Jones, D. B., Jerry, D. R., Wade, N. M., Raadsma, H. W., Huerlimann, R., \& Zenger, K. R. (2018). The state of "Omics" research for farmed penaeids: advances in research and impediments to industry utilization. Frontiers in Genetics, 9, 282. doi:10.3389/fgene.2018.00282

Haas, B. J., Salzberg, S. L., Zhu, W., Pertea, M., Allen, J. E., Orvis, J., . . Wortman, J. R. (2008). Automated eukaryotic gene structure annotation using EVidenceModeler and the program to assemble spliced alignments. Genome Biology, 9(1), R7. doi:10.1186/gb-2008-9-1-r7 
Hoff, K. J., \& Stanke, M. (2019). Predicting genes in single genomes with AUGUSTUS. Current Protocols in Bioinformatics, 65(1), e57. doi:10.1002/cpbi.57

Hu, X., Yang, H.-L., Yan, Y.-Y., Zhang, C.-X., Ye, J.-d., Lu, K.-L., . . Sun, Y.-Z. (2019). Effects of fructooligosaccharide on growth, immunity and intestinal microbiota of shrimp (Litopenaeus vannamei) fed diets with fish meal partially replaced by soybean meal. Aquaculture Nutrition, 25(1), 194-204. doi:10.1111/anu.12843

Huang, S.-W., Lin, Y.-Y., You, E.-M., Liu, T.-T., Shu, H.-Y., Wu, K.-M., . . Yu, H.-T. (2011). Fosmid library end sequencing reveals a rarely known genome structure of marine shrimp Penaeus monodon. BMC Genomics, 12(1), 242. doi:10.1186/1471-2164-12-242

Huerlimann, R., Wade, N. M., Gordon, L., Montenegro, J. D., Goodall, J., McWilliam, S., . . Jerry, D. R. (2018). De novo assembly, characterization, functional annotation and expression patterns of the black tiger shrimp (Penaeus monodon) transcriptome. Scientific Reports, 8(1), 13553. doi:10.1038/s41598-018-31148-4

Huerta-Cepas, J., Szklarczyk, D., Heller, D., Hernández-Plaza, A., Forslund, S. K., Cook, H., . . Bork, P. (2019). eggNOG 5.0: a hierarchical, functionally and phylogenetically annotated orthology resource based on 5090 organisms and 2502 viruses. Nucleic Acids Research, 47(D1), D309-D314. doi:10.1093/nar/gky1085

Jackson, C. J., \& Wang, Y. G. (1998). Modelling growth rate of Penaeus monodon Fabricius in intensively managed ponds: effects of temperature, pond age and stocking density. Aquaculture Research, 29(1), 27-36. doi:10.1111/j.1365-2109.1998.tb01358.x

Jones, D. T., Taylor, W. R., \& Thornton, J. M. (1992). The rapid generation of mutation data matrices from protein sequences. Bioinformatics, 8(3), 275-282. doi:10.1093/bioinformatics/8.3.275

Jones, P., Binns, D., Chang, H.-Y., Fraser, M., Li, W., McAnulla, C., . . Hunter, S. (2014). InterProScan 5: genome-scale protein function classification. Bioinformatics, 30(9), 12361240. doi:10.1093/bioinformatics/btu031

Kanehisa, M., Sato, Y., Kawashima, M., Furumichi, M., \& Tanabe, M. (2016). KEGG as a reference resource for gene and protein annotation. Nucleic Acids Research, 44(D1), D457-462. doi:10.1093/nar/gkv1070

Kao, D., Lai, A. G., Stamataki, E., Rosic, S., Konstantinides, N., Jarvis, E., . . Aboobaker, A. (2016). The genome of the crustacean Parhyale hawaiensis, a model for animal development, regeneration, immunity and lignocellulose digestion. eLife, 5, e20062. doi:10.7554/eLife.20062

Karoonuthaisiri, N., Sittikankeaw, K., Preechaphol, R., Kalachikov, S., Wongsurawat, T., Uawisetwathana, U., ... Kirtikara, K. (2009). ReproArray(GTS): a cDNA microarray for identification of reproduction-related genes in the giant tiger shrimp Penaeus monodon and characterization of a novel nuclear autoantigenic sperm protein (NASP) gene. Comparative Biochemistry and Physiology - Part D: Genomics and Proteomics, 4(2), 90-99. doi:10.1016/j.cbd.2008.11.003

Kent, W. J. (2002). BLAT--the BLAST-like alignment tool. Genome Res, 12(4), 656-664. doi:10.1101/gr.229202

Kiepe, D., Ciarmatori, S., Hoeflich, A., Wolf, E., \& To $\square$ nshoff, B. (2005). Insulin-like growth factor (IGF)-I stimulates cell proliferation and Induces IGF binding protein (IGFBP)-3 and IGFBP-5 gene expression in cultured growth plate chondrocytes via distinct signaling pathways. Endocrinology, 146(7), 3096-3104. doi:10.1210/en.2005-0324

Kim, E. J., Kim, S. J., Park, C. J., \& Nam, Y. K. (2019). Characterization of testis-specific serine/threonine kinase 1-like (TSSK1-like) gene and expression patterns in diploid and triploid Pacific abalone (Haliotis discus hannai; Gastropoda; Mollusca) males. PLoS One, 14(12), e0226022. doi:10.1371/journal.pone.0226022

Koehorst, J. J., van Dam, J. C. J., Saccenti, E., Martins Dos Santos, V. A. P., Suarez-Diez, M., \& Schaap, P. J. (2018). SAPP: functional genome annotation and analysis through a semantic framework using FAIR principles. Bioinformatics, 34(8), 1401-1403. doi:10.1093/bioinformatics/btx767 
Krzywinski, M., Schein, J., Birol, I., Connors, J., Gascoyne, R., Horsman, D., . . Marra, M. A. (2009). Circos: an information aesthetic for comparative genomics. Genome Res, 19(9), 16391645. doi:10.1101/gr.092759.109

Kumar, S., Stecher, G., \& Tamura, K. (2016). MEGA7: Molecular Evolutionary Genetics Analysis Version 7.0 for Bigger Datasets. Molecular Biology and Evolution, 33(7), 1870-1874. doi:10.1093/molbev/msw054

Leelatanawit, R., Uawisetwathana, U., Klinbunga, S., \& Karoonuthaisiri, N. (2011). A cDNA microarray, UniShrimpChip, for identification of genes relevant to testicular development in the black tiger shrimp (Penaeus monodon). BMC Molecular Biology, 12, 15. doi:10.1186/1471-2199-12-15

Lehnert, S. A., Wilson, K. J., Byrne, K., \& Moore, S. S. (1999). Tissue-Specific Expressed Sequence Tags from the Black Tiger Shrimp Penaeus monodon. Mar Biotechnol, 1(5), 465-0476.

Lex, A., Gehlenborg, N., Strobelt, H., Vuillemot, R., \& Pfister, H. (2014). UpSet: Visualization of Intersecting Sets. IEEE Transactions on Visualization and Computer Graphic, 20(12), 19831992. doi:10.1109/TVCG.2014.2346248

Li, H. (2018). Minimap2: pairwise alignment for nucleotide sequences. Bioinformatics, 34(18), 30943100. doi:10.1093/bioinformatics/bty191

Lieberman-Aiden, E., van Berkum, N. L., Williams, L., Imakaev, M., Ragoczy, T., Telling, A., . . . Dekker, J. (2009). Comprehensive mapping of long-range interactions reveals folding principles of the human genome. Science, 326(5950), 289-293. doi:10.1126/science.1181369

Love, M. I., Huber, W., \& Anders, S. (2014). Moderated estimation of fold change and dispersion for RNA-seq data with DESeq2. Genome Biology, 15(12), 550. doi:10.1186/s13059-014-0550-8

Mukai, H. (2003). The structure and function of PKN, a protein kinase having a catalytic domain homologous to that of PKC. The Journal of Biochemistry, 133(1), 17-27. doi:10.1093/jb/mvg019

Oboh, A., Navarro, J. C., Tocher, D. R., \& Monroig, O. (2017). Elongation of very long-chain (>C24) fatty acids in clarias gariepinus: cloning, functional characterization and tissue expression of elov14 elongases. Lipids, 52(10), 837-848. doi:10.1007/s11745-017-4289-3

Olmos, J., Ochoa, L., Paniagua-Michel, J., \& Contreras, R. (2011). Functional feed assessment on Litopenaeus vannamei using $100 \%$ fish meal replacement by soybean meal, high levels of complex carbohydrates and Bacillus probiotic strains. Marine Drugs, 9(6), 1119-1132. doi:10.3390/md9061119

Paradis, E., \& Schliep, K. (2018). ape 5.0: an environment for modern phylogenetics and evolutionary analyses in R. Bioinformatics, 35(3), 526-528. doi:10.1093/bioinformatics/bty633

Pickeral, O. K., Makałowski, W., Boguski, M. S., \& Boeke, J. D. (2000). Frequent human genomic DNA transduction driven by LINE-1 retrotransposition. Genome Res, 10(4), 411-415. doi:10.1101/gr.10.4.411

Pootakham, W., Uengwetwanit, T., Sonthirod, C., Sittikankaew, K., \& Karoonuthaisiri, N. (2020). A novel full-Length transcriptome resource for black tiger shrimp (Penaeus monodon) developed using isoform sequencing (Iso-Seq). Frontiers in Marine Science, 7, 172. doi:10.3389/fmars.2020.00172

Poynton, H. C., Hasenbein, S., Benoit, J. B., Sepulveda, M. S., Poelchau, M. F., Hughes, D. S. T., . . . Richards, S. (2018). The toxicogenome of Hyalella azteca: a model for sediment ecotoxicology and evolutionary toxicology. Environmental Science \& Technology, 52(10), 6009-6022. doi:10.1021/acs.est.8b00837

Putnam, N. H., O'Connell, B. L., Stites, J. C., Rice, B. J., Blanchette, M., Calef, R., . . Green, R. E. (2016). Chromosome-scale shotgun assembly using an in vitro method for long-range linkage. Genome Res, 26(3), 342-350. doi:10.1101/gr.193474.115

Quyen, D. V., Gan, H. M., Lee, Y. P., Nguyen, D. D., Nguyen, T. H., Tran, X. T., . . Austin, C. M. (2020). Improved genomic resources for the black tiger prawn (Penaeus monodon). Marine Genomics, 100751. 
R Core Team. (2019). R: a language and environment for statistical computing. Vienna, Austria: R Foundation for Statistical Computing. Retrieved from https://www.R-project.org/

Reference standard for genome biology. (2018). A reference standard for genome biology. Nature Biotechnology, 36(12), 1121-1121. doi:10.1038/nbt.4318

Sagar, V., Sahu, N. P., Pal, A. K., Hassaan, M., Jain, K. K., Salim, H. S., . . El-Haroun, E. R. (2019). Effect of different stock types and dietary protein levels on key enzyme activities of glycolysis-gluconeogenesis, the pentose phosphate pathway and amino acid metabolism in Macrobrachium rosenbergii. Journal of Applied Ichthyology, 35(4), 1016-1024. doi:10.1111/jai.13931

Santos, C. A., Andrade, S. C. S., Teixeira, A. K., Farias, F., Kurkjian, K., Guerrelhas, A. C., . . Freitas, P. D. (2018). Litopenaeus vannamei transcriptome profile of populations evaluated for growth performance and exposed to white spot syndrome virus (WSSV). Frontiers in Genetics, 9(120). doi:10.3389/fgene.2018.00120

Schrader, L., \& Schmitz, J. (2019). The impact of transposable elements in adaptive evolution. Molecular Ecology, 28(6), 1537-1549. doi:10.1111/mec.14794

Shi, Y., \& He, M.-x. (2016). PfIRR Interacts with HrIGF-I and activates the MAP-kinase and PI3kinase signaling pathways to regulate glycogen metabolism in Pinctada fucata. Scientific Reports, 6(1), 22063. doi:10.1038/srep22063

Simão, F. A., Waterhouse, R. M., Ioannidis, P., Kriventseva, E. V., \& Zdobnov, E. M. (2015). BUSCO: assessing genome assembly and annotation completeness with single-copy orthologs. Bioinformatics, 31, 3210-3212.

Sittikankaew, K., Pootakham, W., Sonthirod, C., Sangsrakru, D., Yoocha, T., Khudet, J., . . . Karoonuthaisiri, N. (2020). Transcriptome analyses reveal the synergistic effects of feeding and eyestalk ablation on ovarian maturation in black tiger shrimp. Scientific Reports, 10(1), 3239. doi:10.1038/s41598-020-60192-2

Slater, G. S. C., \& Birney, E. (2005). Automated generation of heuristics for biological sequence comparison. BMC Bioinform, 6(1), 31. doi:10.1186/1471-2105-6-31

Smit, A., Hubley, R., \& Green, P. (2013-2015). RepeatMasker Open-4.0. http://www.repeatmasker.org.

Snipen, L., \& Ussery, D. W. (2010). Standard operating procedure for computing pangenome trees. Standards in Genomic Sciences, 2(1), 135-141. doi:10.4056/sigs.38923

Stanke, M., Diekhans, M., Baertsch, R., \& Haussler, D. (2008). Using native and syntenically mapped cDNA alignments to improve de novo gene finding. Bioinformatics, 24(5), 637-644. doi:10.1093/bioinformatics/btn013

Steinegger, M., \& Söding, J. (2017). MMseqs2 enables sensitive protein sequence searching for the analysis of massive data sets. Nature Biotechnology, 35(11), 1026-1028. doi:10.1038/nbt.3988

Supungul, P., Klinbunga, S., Pichyangkura, R., Jitrapakdee, S., Hirono, I., Aoki, T., \& Tassanakajon, A. (2002). Identification of immune-related genes in hemocytes of black tiger shrimp (Penaeus monodon). Mar Biotechnol, 4(5), 487-494. doi:10.1007/s10126-002-0043-8

Swathi, A., Shekhar, M. S., Katneni, V. K., \& Vijayan, K. K. (2018). Genome size estimation of brackishwater fishes and penaeid shrimps by flow cytometry. Molecular Biology Reports, 45(5), 951-960. doi:10.1007/s11033-018-4243-3

Tassanakajon, A., Klinbunga, S., Paunglarp, N., Rimphanitchayakit, V., Udomkit, A., Jitrapakdee, S., . .. Lursinsap, C. (2006). Penaeus monodon gene discovery project: The generation of an EST collection and establishment of a database. Gene, 384, 104-112. doi:10.1016/j.gene.2006.07.012

Tassanakajon, A., Pongsomboon, S., Jarayabhand, P., Klinbunga, S., \& Boonsaeng, V. V. (1998). Genetic structure in wild populations of black tiger shrimp (Penaeus monodon) using randomly amplified polymorphic DNA analysis. J Mar Biotechnol, 6(4), 249-254.

Tassanakajon, A., Pongsomboon, S., Rimphanitchayakit, V., Jarayabhand, P., \& Boonsaeng, V. (1997). Random amplified polymorphic DNA (RAPD) markers for determination of genetic 
variation in wild populations of the black tiger prawn (Penaeus monodon) in Thailand. Mol Mar Biol Biotechnol, 6(2), 110-115.

Thornber, K., Verner-Jeffreys, D., Hinchliffe, S., Rahman, M. M., Bass, D., \& Tyler, C. R. (2019). Evaluating antimicrobial resistance in the global shrimp industry. Reviews in Aquaculture, 121. doi:10.1111/raq. 12367

Toledo, T. M., Silva, B. C., Vieira, F. d. N., Mouriño, J. L. P., \& Seiffert, W. Q. (2016). Effects of different dietary lipid levels and fatty acids profile in the culture of white shrimp Litopenaeus vannamei (Boone) in biofloc technology: water quality, biofloc composition, growth and health. Aquac Res, 47(6), 1841-1851. doi:10.1111/are.12642

Tong, J., Lehnert, S. A., Byrne, K., Kwan, H. S., \& Chu, K. H. (2002). Development of polymorphic EST markers in Penaeus monodon: applications in penaeid genetics. Aquaculture, 208(1-2), 69-79. doi:10.1016/S0044-8486(01)00706-2

Tørresen, O. K., Star, B., Mier, P., Andrade-Navarro, M. A., Bateman, A., Jarnot, P., . . Linke, D. (2019). Tandem repeats lead to sequence assembly errors and impose multi-level challenges for genome and protein databases. Nucleic Acids Research, 47(21), 10994-11006. doi:10.1093/nar/gkz841

Turkmen, S., Perera, E., Zamorano, M. J., Simó-Mirabet, P., Xu, H., Pérez-Sánchez, J., \& Izquierdo, M. (2019). Effects of dietary lipid composition and fatty acid desaturase 2 expression in broodstock gilthead sea bream on lipid metabolism-related genes and methylation of the fads 2 gene promoter in their Offspring. International Journal of Molecular Sciences, 20(24), 6250. doi:10.3390/ijms20246250

Uengwetwanit, T., Ponza, P., Sangsrakru, D., Wichadakul, D., Ingsriswang, S., Leelatanawit, R., . . . Karoonuthaisiri, N. (2018). Transcriptome-based discovery of pathways and genes related to reproduction of the black tiger shrimp (Penaeus monodon). Marine Genomics, 37, 69-73. doi:10.1016/j.margen.2017.08.007

Watts, J. L., \& Browse, J. (2006). Dietary manipulation implicates lipid signaling in the regulation of germ cell maintenance in C. elegans. Developmental Biology, 292(2), 381-392. doi:10.1016/j.ydbio.2006.01.013

Wilson, K., Cahill, V., Ballment, E., \& Benzie, J. (2000). The complete sequence of the mitochondrial genome of the crustacean Penaeus monodon: Are malacostracan crustaceans more closely related to Insects than to branchiopods? Molecular Biology and Evolution, 17(6), 863-874. doi:10.1093/oxfordjournals.molbev.a026366

Wilson, K., Li, Y., Whan, V., Lehnert, S., Byrne, K., Moore, S., . . Benzie, J. (2002). Genetic mapping of the black tiger shrimp Penaeus monodon with amplified fragment length polymorphism. Aquaculture, 204(3-4), 297-309. doi:10.1016/S0044-8486(01)00842-0

Wongsurawat, T., Leelatanawit, R., Thamniemdee, N., Uawisetwathana, U., Karoonuthaisiri, N., Menasveta, P., \& Klinbunga, S. (2010). Identification of testis-relevant genes using in silico analysis from testis ESTs and cDNA microarray in the black tiger shrimp (Penaeus monodon). BMC Molecular Biology, 11, 55. doi:10.1186/1471-2199-11-55

Wu, T. D., \& Watanabe, C. K. (2005). GMAP: a genomic mapping and alignment program for mRNA and EST sequences. Bioinformatics, 21(9), 1859-1875. doi:10.1093/bioinformatics/bti310

Wuthisuthimethavee, S., Aoki, T., Hirono, I., \& Tassanakajon, A. (2009). Preliminary study of BAC library construction in black tiger shrimp, Penaeus monodon. Walailak Journal of Science and Technology, 6(1), 37-48.

Yu, Y., Zhang, X., Yuan, J., Li, F., Chen, X., Zhao, Y., . . Xiang, J. (2015). Genome survey and highdensity genetic map construction provide genomic and genetic resources for the Pacific White Shrimp Litopenaeus vannamei. Scientific Reports, 5(1), 15612. doi:10.1038/srep15612

Yuan, J., Zhang, X., Liu, C., Yu, Y., Wei, J., Li, F., \& Xiang, J. (2018). Genomic resources and comparative analyses of two economical penaeid shrimp species, Marsupenaeus japonicus and Penaeus monodon. Marine Genomics, 39, 22-25. doi:10.1016/j.margen.2017.12.006 
Zhang, X., Yuan, J., Sun, Y., Li, S., Gao, Y., Yu, Y., . . Xiang, J. (2019). Penaeid shrimp genome provides insights into benthic adaptation and frequent molting. Nat Commun, 10(1), 356. doi:10.1038/s41467-018-08197-4

Zhao, C., Zhang, X., Liu, C., Huan, P., Li, F., Xiang, J., \& Huang, C. (2012). BAC end sequencing of Pacific white shrimp Litopenaeus vannamei: a glimpse into the genome of Penaeid shrimp. Chinese Journal of Oceanology and Limnology, 30(3), 456-470. doi:10.1007/s00343-012$1159-\mathrm{y}$

\section{Data accessibility}

The genomic and transcriptomic data are available in the NCBI under the following accession numbers: PRJNA611030 and PRJNA602748. The annotation file and genome sequences are available from the following website: http://www.biotec.or.th/pmonodon/index.php.

\section{Author contributions}


bioRxiv preprint doi: https://doi.org/10.1101/2020.05.14.096073; this version posted May 16, 2020. The copyright holder for this preprint (which

was not certified by peer review) is the author/funder, who has granted bioRxiv a license to display the preprint in perpetuity. It is made available under aCC-BY-NC-ND 4.0 International license.

877 Table 1. Assembly statistics of the $P$. monodon genome.

\begin{tabular}{lrrr}
\hline & PacBio & PacBio+Chicago & $\begin{array}{r}\text { PacBio+Chicago } \\
\text { +Hi-C }\end{array}$ \\
\hline Number of contigs/scaffolds & 70,380 & 30,179 & 26,877 \\
Number of contigs/scaffolds $>\square 1 \square \mathrm{kb}$ & 70,373 & 30,171 & 26,869 \\
Number of contigs/scaffolds $>\square 50 \square \mathrm{kb}$ & 11,084 & 3,725 & 869 \\
Number of contigs/scaffolds $>1 \mathrm{Mb}$ & 6 & 569 & 44 \\
Number of contigs/scaffolds $>10 \mathrm{Mb}$ & - & 1 & 43 \\
Total length (bases) & $2,389,954,102$ & $2,394,031,700$ & $2,394,363,600$ \\
Longest contig/scaffold (bases) & $1,387,722$ & $12,098,070$ & $65,869,259$ \\
Mean contig/scaffold size (bases) & 33,958 & 79,328 & 89,086 \\
Contig/scaffold N50 (Mb) & 0.079 & 1.157 & 44.862 \\
Contig/scaffold L50 & 6,786 & 509 & 23 \\
\% N in scaffolds & - & 0.17 & 0.18 \\
\hline Genome annotation & & Number of genes & 31,641 \\
& Number of transcripts & 36,538 \\
& Average gene length (bp) & 1,428 \\
& Number of annotated genes: & Refseq \\
& & Uniprot & 30,038 \\
& Go & 10,068 \\
& & GnterPro & 22,243 \\
& & & 20,615 \\
\hline
\end{tabular}

Figure 1. P. monodon genome assembly and phylogenetic analysis.

(A) Genomic landscape of 44 assembled $P$. monodon chromosomes. (a) Physical map of $P$. monodon chromosomes (Mb scale). (b) Density of repetitive sequences represented by percentage of genomic regions covered by simple repeat sequences in 500-kb window. (c) Gene density represented by number of genes in 500-kb window. (d) GC content represented by percentage of G/C bases in 500$\mathrm{kb}$ window. Syntenic blocks are depicted by connected lines. (e) Syntenic relationship of gene blocks among $P$. monodon chromosomes. Syntenic blocks were identified by MCScanX with criteria at least ten syntenic genes and a maximum of six intervening genes allowed. (B) Diagrams showing colinearity between P. monodon and L. vannamei chromosomes. Lines link the position of orthologous gene sets. Most regions exhibit one-to-one relationship between $P$. monodon and $L$. vannamei. The yellow line represents the region on a single $P$. monodon chromosome that exhibits synteny to regions on two L. vannamei chromosomes. Black tiger shrimp chromosomes are designated with "P" followed by chromosome numbers, and Pacific white shrimp chromosomes are designated with "L" followed by the chromosome numbers. (C) Diagrams showing colinearity between $P$. monodon and L. vannamei where the syntenic relationship between chromosomes is not one-to-one. (D) Phylogenetic tree of concatenated mitochondria protein-coding genes using maximum likelihood general reversible mitochondrial model. The percentage of trees in which the associated taxa clustered together is shown next to the branches. The tree is drawn to scale, with branch lengths measured in the number of substitutions per site.

Figure 2. Comparative genomics and repeat elements analysis. 
(A) Upset plot represents pan-core protein family of crustacean genomes (P. monodon, L. vannamei, $P$. hawaiensis, E. affinis, H. azteca, and D. pulex) with the pan protein family tree with bootstrap value in black numbers. The numbers on branches indicate the number of protein family gains (green) or losses (red). Relative proportion or repeat elements in the crustacean genomes is presented as (B) bar plots of percentage of repeat elements and $(\mathbf{C})$ relative abundance of each LINE type.

Figure 3. Expression of differentially expressed genes of slow-growing shrimp $(n=15)$ and fastgrowing shrimp $(\mathbf{n}=15)$.

(A) The top five enriched COGs metabolism groups were carbohydrate transport and metabolism, secondary metabolites biosynthesis, transport and catabolism, inorganic ion transport and metabolism, lipid transport and metabolism and amino acid transport and metabolism. (B) Differentially expressed genes in PI3K-Akt pathway. Blue letters indicate newly identified genes in P. monodon, and the dagger $(\dagger)$ indicates genes that were further validated by quantitative real-time PCR. 
A

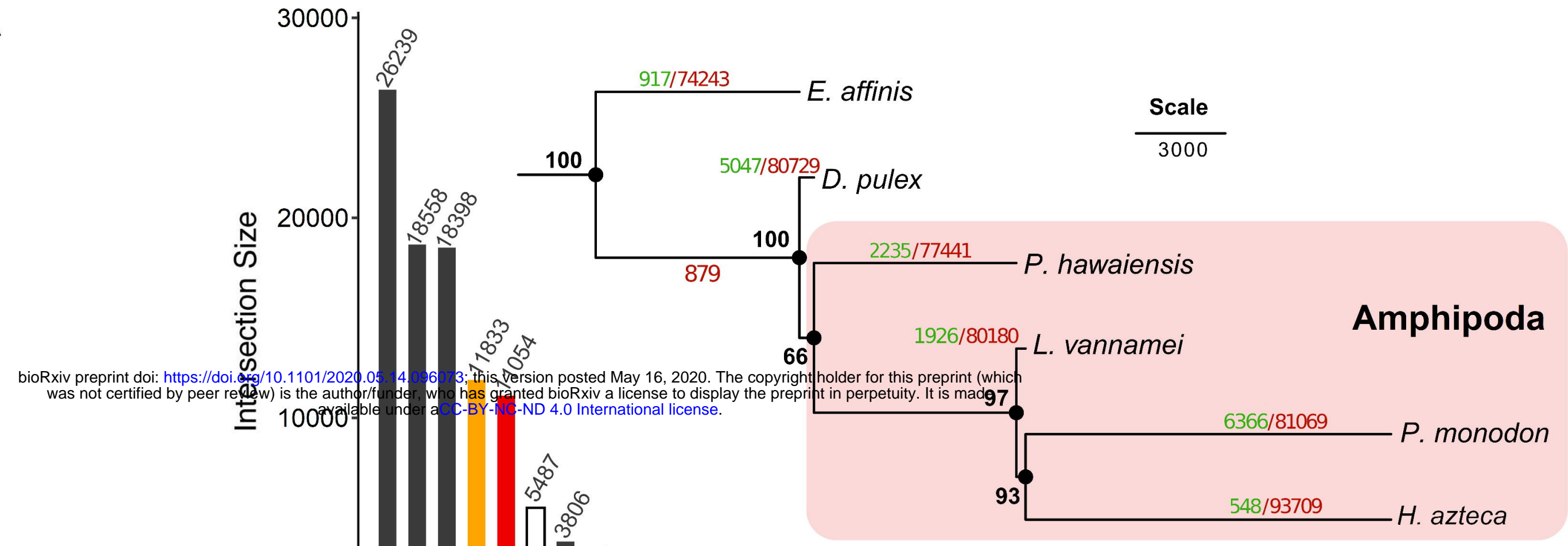

Protein family size

H. azteca P. monodon L. vannamei D. pulex $P$. hawaiensis E. affinis

$20,000 \quad 10,000 \quad 0$

B
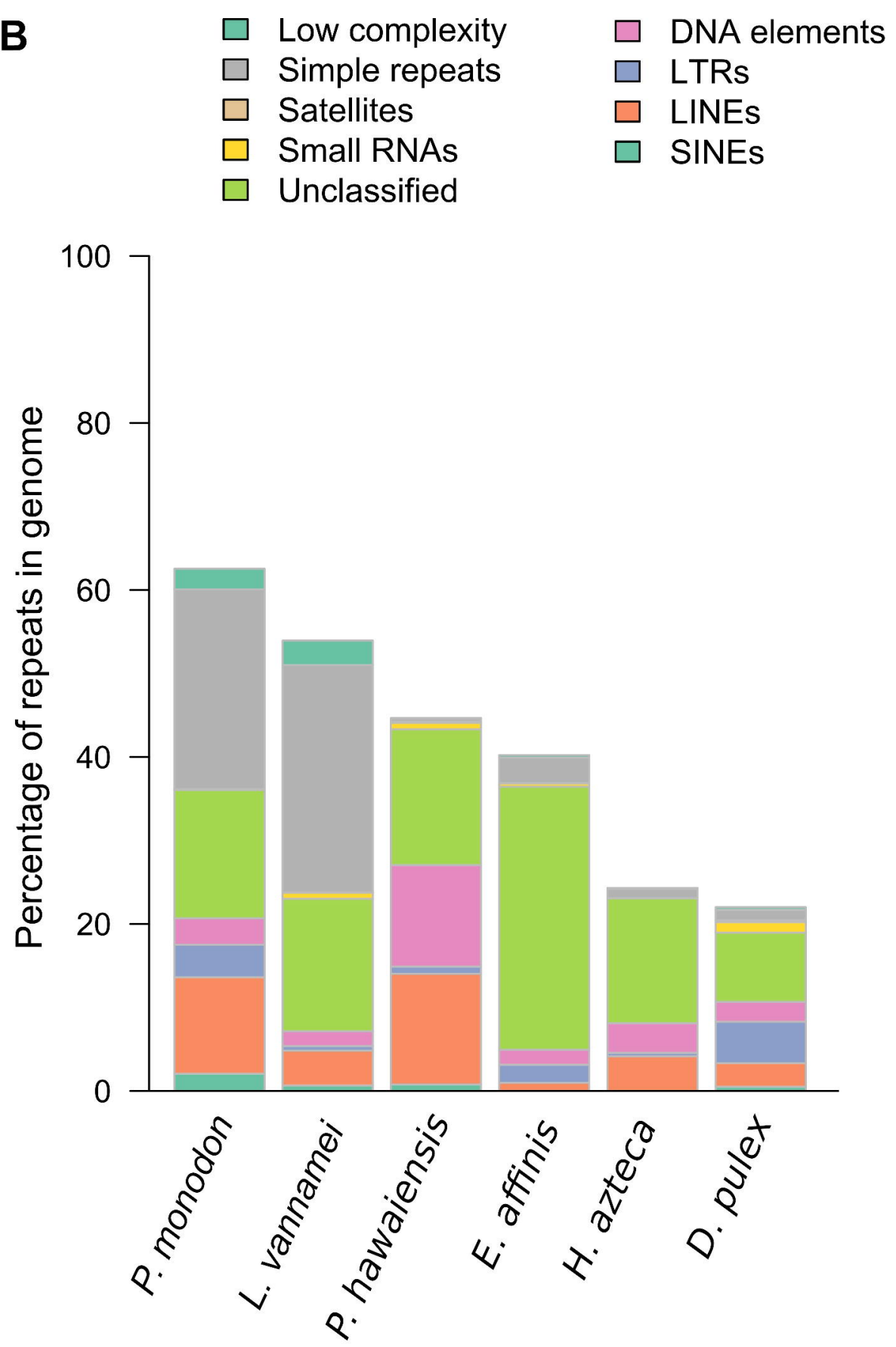
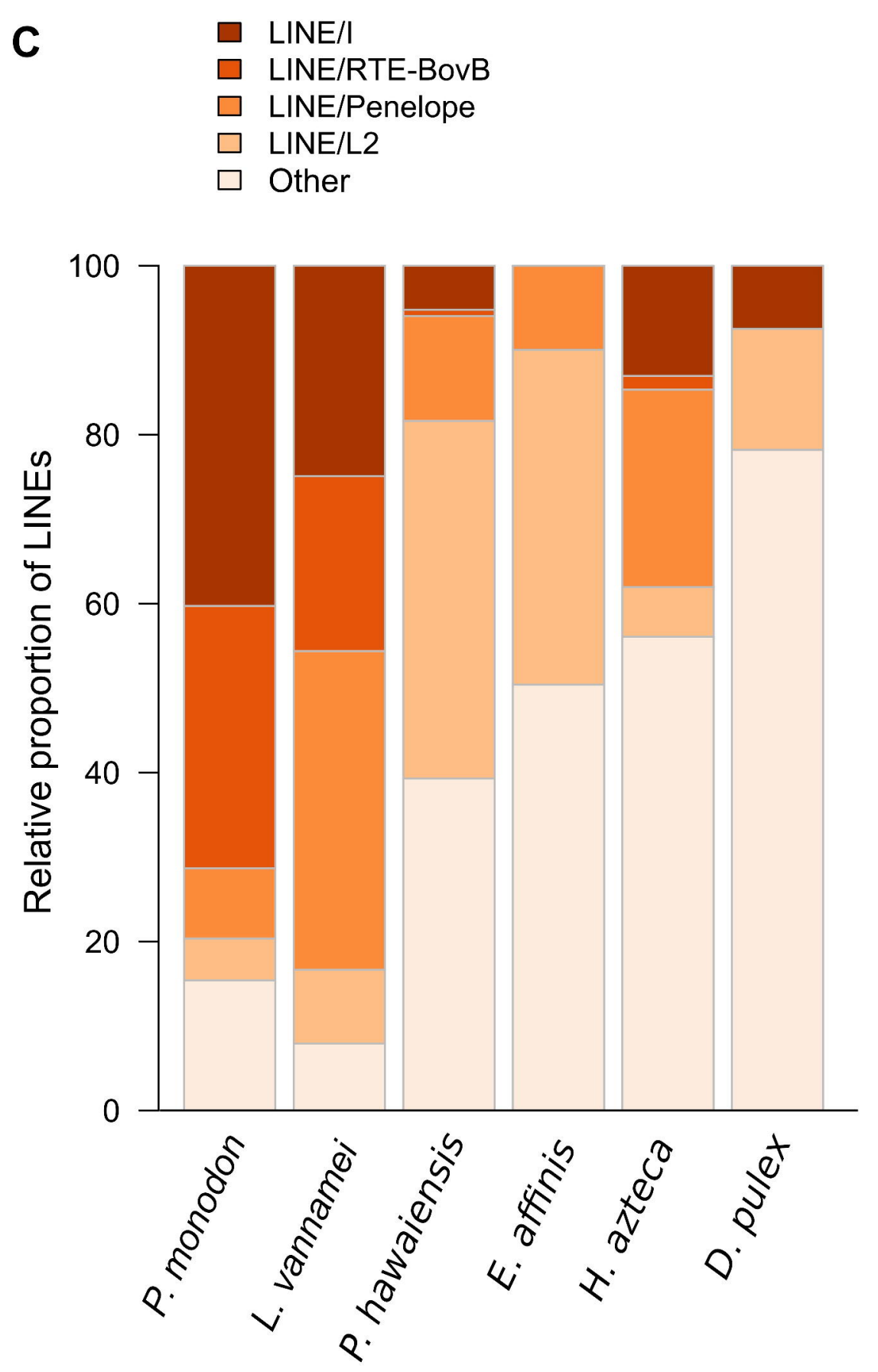
+ glycerol-3-phosphate acyltransferase 3 isoform X2

1.54 acyl-CoA Delta(11) desaturase-like

1.43 fatty acid hydroxylase domain-containing protein 2 isoform $X 1$

lipase member $\mathrm{H}$

putative acyl-coenzyme A oxidase 3.2 , peroxisomal

G-protein coupled receptor Mth2-like

pancreatic lipase-related protein 2-like

cellular retinoic acid-binding protein 1-like

long-chain-fatty-acid--CoA ligase 4 isoform $\times 1$

non-specific lipid-transfer protein

sodium/calcium exchanger regulatory protein 1-like

lipase 3-like

\begin{tabular}{|l|l|l|l}
\hline & -1.04 & AMP-binding protein \\
\hline & -1.11 & beta-1,3-glucan-binding protein precursor \\
& -1.22 & 4-coumarate--CoA ligase 1-like \\
& -1.60 & elongation of very long chain fatty acids protein 4-like \\
& -2.08 & apolipophorins-like \\
\hline
\end{tabular}

-2.08 apolipophorins-like

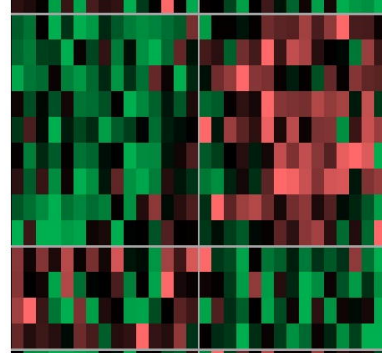

2.10 xaa-Pro aminopeptidase 1-like

1.62 RNA-directed DNA polymerase from mobile element jockey-like

1.38 cystathionine beta-synthase-like isoform $\mathrm{X} 1$

$1.29 \mathrm{~N}$-fatty-acyl-amino acid synthase/hydrolase PM20D1

1.28 arginase-2, mitochondrial

1.20 + phospholipid phosphatase 2-like

1.15 agmatinase, mitochondrial

1.11 phenoloxidase 2

1.02 kynurenine formamidase isoform $\mathrm{X} 1$

-1.06 phosphoserine phosphatase isoform $\mathrm{X} 2$

-1.17 methionine synthase-like

-1.48 betaine--homocysteine S-methyltransferase 1-like

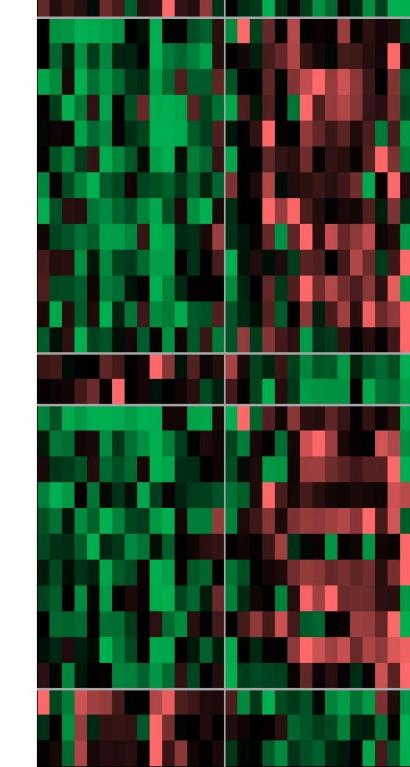

$1.71 \mathrm{Y}+\mathrm{L}$ amino acid transporter 2

2.24 laccase- 4 isoform $X 2$

1.94 cytochrome P450 9e2-like isoform X3

1.82 cytochrome P450 2L1-like

1.44 sorbitol dehydrogenase

1.35 estradiol 17-beta-dehydrogenase 8-like

1.33 cytochrome P450 3A24-like isoform X3

1.25 multidrug resistance-associated protein 1 isoform $X 4$

1.19 probable cytochrome P450 49a1

1.14 estradiol 17-beta-dehydrogenase 8

1.12 guanine deaminase-like

1.11 short-chain dehydrogenase/reductase family $16 \mathrm{C}$ member 6-like

1.10 probable cytochrome P450 49a1

1.07 cytochrome P450 2B4-like

-1.07 ATP-binding cassette sub-family B member 8 , mitochondrial-like

-1.55 retinol dehydrogenase 13 -like

2.93 t transferrin-like

1.85 bestrophin homolog 17 -like isoform $\mathrm{X} 1$

1.64 zinc transporter ZIP1-like

1.59 sodium/hydrogen exchanger 7 isoform $X 5$

1.20 soma ferritin-like

1.18 innexin inx2

1.14 innexin inx2-like

1.13 facilitated trehalose transporter Tret1-like

1.09 sodium-dependent phosphate transporter 2

1.04 innexin shaking-B-like

1.02 probable glutamate receptor

$-1.19+$ sodium/hydrogen exchanger 2 isoform $X$

-1.63 excitatory amino acid transporter 1 -like

-2.04 prestin-like isoform $\times 2$ 